\title{
Botulinum toxin in the treatment of intestinal pseudo-obstruction following a stroke
}

\author{
Przemysław Nowak ${ }^{1}$, Agnieszka Gala-Błądzińska ${ }^{2}$, Katarzyna Stybel ${ }^{2}$, Rafał Filip ${ }^{3}$ \\ ${ }^{1}$ Medical Care Centre, Jaroslaw, Poland \\ ${ }^{2}$ Department of Internal Diseases, Nephrology and Endocrinology, Clinical Hospital No.2, University of Rzeszow, Rzeszow, Poland \\ ${ }^{3}$ Department of Gastroenterology with IBD Unit, Clinical Hospital No.2, University of Rzeszow, Rzeszow, Poland
}

Key words: botulinum toxin, constipation, external anal sphincter, spasticity

(Neurol Neurochir Pol 2020; 54 (6): 589-590)

\section{To the Editors:}

Botulinum toxin type $\mathrm{A}$ is a biochemical neuromodulator known to block cholinergic transmission at the neuromuscular junction. Over the last two decades, the localised efficacy of botulinum toxin $\mathrm{A}$, and its well-tolerated safety profile, have resulted in a significant growth of its on- and off-label use across multiple therapeutic and aesthetic indications [1]. It has also been shown to be effective in the gastrointestinal tract [2-4]. Until now, injections of botulinum toxin have been used in the treatment of, inter alia, chronic idiopathic constipation in children, anal fissure both in children and adults, as well as obstructive symptoms in children with Hirschsprung Disease [5-7]. Botulinum toxin injections are also effective in short-term anismus, defined as a functional disorder of the defecation process that entails relaxation failure or even paradoxical contraction of the puborectalis muscle and contraction of the external anal sphincter during defecation [8]. Zhang et al. evaluated the efficacy of botulinum toxin type $\mathrm{A}$ injection to the puborectalis and external sphincter muscle in the treatment of 31 patients in whom previous biofeedback therapy had proved unsatisfactory [9]. Chronic constipation scoring system and anorectal manometry showed that the injection of botulinum toxin, combined with pelvic floor biofeedback training, was effective in 24 of the 31 patients [9].

\section{Case report}

In November 2018, during post-stroke rehabilitation, a 64-year-old female patient was transferred to the Internal Medicine Department due to severe abdominal distension with symptoms of large bowel obstruction. The previous month, she had suffered a stroke to the left hemisphere, was treated with thrombolysis and thrombectomy, but was left hemiplegic. A differential diagnosis was performed to exclude malignant obstructing diseases or benign conditions such as sigmoid volvulus, diverticulitis or colonic ischaemia. Abdominal and pelvic CT with contrast revealed the persistence of a large faecal impaction located in the sigmoid colon and a massive dilatation from the caecum to the rectum, with transverse colon of up to approximately $75 \mathrm{~mm}$ [normal value: $<60 \mathrm{~mm}$ ] [10], sigmoid colon of up to $100 \mathrm{~mm}$ [normal value: $<65 \mathrm{~mm}$ ] [10] and rectal ampulla of up to $58 \mathrm{~mm}$ [normal value: $<40 \mathrm{~mm}$ ] [10] (Fig. 1). The patient was diagnosed with megacolon in the course of anal spasticity secondary to the stroke. Although the patient had never previously suffered from chronic constipation, at the time of admission she had a complex medical history including hypertension, atrial fibrillation, dehydration and hypopotassemia. Laboratory tests, including blood morphology, CRP and Clostitdium diff., were negative. Physical examination found no signs of fever or tachycardia. Multiple laxatives, enemas and prokinetic agents (e.g. metoclopramide) were introduced. However, no clinical improvement was noted. Pulsed irrigation and abdominal massage yielded only a slight, transient effect. Therefore, a colonic-rectal tube was inserted. The expulsion of a large volume of gas led to significant, but only transient, relief of the abdominal distension and reduction of abdominal symptoms.

After six days of conservative treatment, the abdomen remained visibly distended, and the patient's general condition had deteriorated. Moreover, her CRP level had increased to $25.6 \mathrm{mg} / \mathrm{l}$ [normal value: < 5.0], and white blood cell count elevated to $16 \times 10^{9} / \mathrm{L}$. Surgical consultations confirmed 


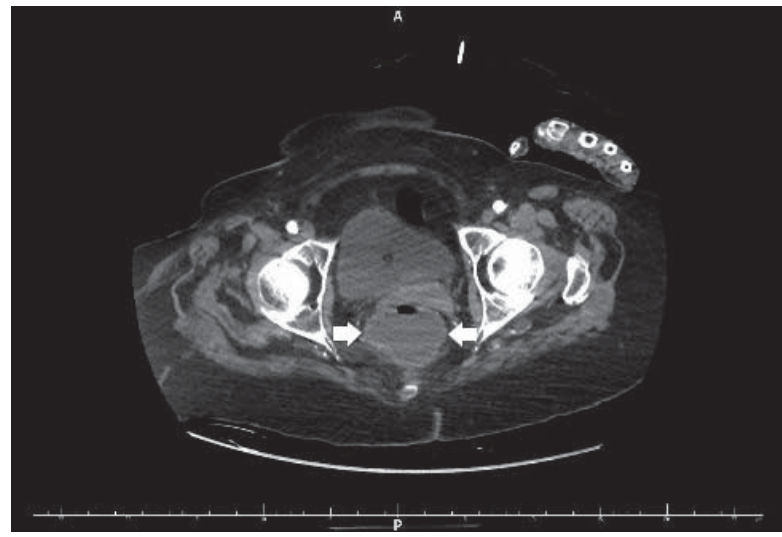

Figure 1. Abdominal and pelvic CT with contrast. Arrows indicate dilatation of rectal ampulla

a redundant megacolon with a high risk of bowel wall ischaemia or/and perforation. Therefore, an elective colectomy was scheduled. However, due to the need to treat coexisting conditions, the surgery was postponed. A radiographic examination at the beginning of January 2019 revealed a colonic dilatation of up to $130 \mathrm{~mm}$ and rectal dilatation of up to $51 \mathrm{~mm}$ (Suppl. Fig. 2). Since several per-rectum examinations revealed anal spasm, the patient was proposed incobotulinumtoxinA injection in the external anal sphincter as a bridge therapy.

\section{Intervention}

At the end of January 2019, the patient in the lithotomy position was injected with $70 \mathrm{U}$ of incobotulinumtoxinA diluted in $2 \mathrm{ml}$ of saline into the external anal sphincter. Injections were administered unilaterally, either via the intra-anal or perianal region ( $35 \mathrm{U}$ on each side). The patient was discharged four days after the initial admission. On discharge, the abdomen remained only slightly distended. The patient was passing 4-8 loose stools and not vomiting. An X-ray examination given four days after the injections revealed colonic dilatation of up to $120-130 \mathrm{~mm}$ and rectal dilatation of up to $34 \mathrm{~mm}$ (Suppl. Fig. 3). The patient was discharged from the ward. Two weeks following the procedure, a per-rectum examination showed decreased sphincter spasticity, as well as a significant, noticeable reduction of abdominal symptoms. Although control of the anal sphincter was not fully regained, reduced constipation led to a remarkable improvement in the quality of life.

The procedure was repeated after three months, in April 2019. Due to the improvement of the patient's condition, the dose of incobotulinumtoxinA was lowered to $50 \mathrm{U}$ ( $25 \mathrm{U}$ on each side) to prevent excessive external anal sphincter relaxation. The same technique of injection was applied. Two weeks after this administration, the patient had partially regained control of the anal sphincter.

It is important to note that the two-month rehabilitation (June-July 2019) did not influence the overall neurological condition. Kinesitherapy did not significantly improve the patient's motor function, and continuous logotherapy led to only partial recovery from aphasia. After rehabilitation, hemiplegia turned into hemiparesis, and the patient was discharged in a wheelchair.

After another six months, in October 2019, the therapeutic effect of injections was sustained. The patient regained full control of the external anal sphincter and controlled defecation herself. A lower gastrointestinal series examination revealed the width of the rectal ampulla at $36 \mathrm{~mm}$ (Suppl. Fig. 4).

In this case report, we demonstrate the potential of botulinum toxin in the treatment of external anal sphincter spasm. An initial 'rescue' local injection of BoNT-A not only allowed surgical decompression of the GI tract to be avoided, but over the longer term also led to the regaining of full control of defecation in a post-stroke patient.

In conclusion, injections of BoNT-A may be a useful alternative treatment modality for post-stroke constipation, especially for patients who do not respond well to conventional therapy.

\section{References}

1. Fonfria E, Maignel J, Lezmi S, et al. The Expanding Therapeutic Utility of Botulinum Neurotoxins. Toxins (Basel). 2018; 10(5), doi: 10.3390/ toxins10050208, indexed in Pubmed: 29783676.

2. Brisinda G, Sivestrini N, Bianco G, et al. Treatment of gastrointestinal sphincters spasms with botulinum toxin A. Toxins (Basel). 2015; 7(6): 1882-1916, doi: 10.3390/toxins 7061882, indexed in Pubmed: 26035487.

3. Madaliński MH, Sławek J, Duzyński W, et al. Side effects of botulinum toxin injection for benign anal disorders. European Journal of Gastroenterology \& Hepatology. 2002;14. ; 853: 856.

4. Madalinski MH, Slawek J, Zbytek B, et al. Topical nitrates and the higher doses of botulinum toxin for chronic anal fissure. Hepatogastroenterology. 2001; 48(40): 977-979, indexed in Pubmed: 11490852.

5. Ahmadi J, Azary S, Ashjaei B, et al. Intrasphincteric botulinum toxin injection in treatment of chronic idiopathic constipation in children. Iran J Pediatr. 2013; 23(5): 574-578, indexed in Pubmed: 24800020.

6. Wester T, Granström AL. Botulinum toxin is efficient to treat obstructive symptoms in children with Hirschsprung disease. Pediatr Surg Int. 2015; 31(3): 255-259, doi: 10.1007/s00383-015-3665-4, indexed in Pubmed: 25616563.

7. Wald A, Bharucha AE, Cosman BC, et al. ACG clinical guideline: management of benign anorectal disorders. Am J Gastroenterol. 2014; 109(8): 1141-57; (Quiz) 1058, doi: 10.1038/ajg.2014.190, indexed in Pubmed: 25022811.

8. Emile SH, Elfeki HA, Elbanna HG, et al. Efficacy and safety of botulinum toxin in treatment of anismus: A systematic review. World J Gastrointest Pharmacol Ther. 2016; 7(3): 453-462, doi: 10.4292/ wjgpt.v7.i3.453, indexed in Pubmed: 27602248.

9. Zhang Y, Wang ZN, He L, et al. Botulinum toxin type-A injection to treat patients with intractable anismus unresponsive to simple biofeedback training. World J Gastroenterol. 2014; 20(35): 12602-12607, doi: 10.3748/wgg.v20.i35.12602, indexed in Pubmed: 25253964.

10. Cuda T, Gunnarsson R, de Costa A. Symptoms and diagnostic criteria of acquired Megacolon - a systematic literature review. BMC Gastroenterol. 2018; 18(1): 25, doi: 10.1186/s12876-018-0753-7, indexed in Pubmed: 29385992. 